\title{
Malaria parasites of lemurs
}

\author{
by P. C. C. GARNHAM * and G. UILENBERG ** \\ * Imperial College of Science and Technology, \\ Ashurst Lodge, Ascot, Berks., England. \\ **Food and Agriculture Organisation of the United Nations \\ Box 9182, Dar es Salaam, Tanzania.
}

\section{Summary.}

Four species of Plasmodium have been described from lemurs: P. girardi, P. foleyi, $P$. lemuris and an unnamed species. $P$. girardi is redescribed and the other three species are here shown to be identical in essential features. $P$. foleyi is accordingly redescribed and the name, $P$. lemuris, becomes a synonym.

\section{Résumé.}

Plasmodium spp. chez les Lémuriens.

Quatre espèces de Plasmodium ont été signalées chez les lémuriens : P. girardi, P. foleyi, $P$. lemuris et une espèce anonyme. $P$. girardi est redécrit et les autres trois espèces sont comparables par tous leurs principaux caractères. Ainsi, $P$. foleyi est redécrit et le nom $P$. lemuris tombe en synonymie.

Three plasmodial taxa have been described from lemurs, Plasmodium girardi and $P$. foleyi by Bück et al. in 1952 and $P$. lemuris by Huff and Hoogstraal in 1963. The status of $P$. foleyi was regarded by Garnham (1966) as questionable; its characters suggested that it might belong to the Haemoproteidae rather than to the Plasmodiidae. Only 30 examples of $P$. lemuris were found in the single blood smear available, in which not all stages of development of the parasite were seen.

Since 1966 one of us (G.U.) has obtained further and more abundant material from lemurs, and after prolonged study we have reached a conclusion on the nature of these interesting organisms. This latest material exhibited certain differences from the morphology of the named species. The problem therefore was to decide on the relative position of the four possible species : Plasmodium girardi, Plasmodium (incertae sedis) foleyi, Plasmodium lemuris and the Plasmodium sp. discovered by Uilenberg (1970). 


\section{Plasmodium girardi}

Although our observations on the morphology of $P$. girardi do not agree in all particulars with the original description of Bück et al. (1952), the resemblances are sufficiently close to enable us to conclude that we were studying the same species. This opinion is strengthened by the presence in both series, viz. Bück's et al. and ours, of certain highly characteristic features. Our data are derived from 2 naturally infected animals (L. macaco macaco and L. m. fulvus), studied over a period of several months, and in view of this additional information we present here a redescription of $P$. girardi Bück et al., 1952, as seen in the blood of splenectomised lemurs. A Lemur mongoz mongoz was splenectomised and inoculated with infected blood; 13 days later it showed parasites in the blood and these reached a peak after a further week, when parasitaemia sank to a low level. L. catta is apparently insusceptible, but only a single inoculation was performed, and observations were made for only 23 days.

Earliest forms and trophozoites. The youngest stages (fig. $1 A-B$ ) appear to be merozoites (about $1.5 \mu \mathrm{m}$ in diameter) which have just entered the corpuscule, and large numbers of these parasites and slightly older forms were present in the blood films. They consist chiefly of a large irregular nucleus and a wisp of cytoplasm. The merozoite grows into an ill-formed ring with a small vacuole and measures about $2 \mu \mathrm{m}$, By the time it has reached a diameter of $3 \mu \mathrm{m}$, one or two black dots of pigment (fig. $1 C-D$ ) appear in the cytoplasm. Multiple infections of the erythrocyte are uncommon, but up to four parasites in a single cell have been seen. Accote forms are uncommon. Although the young forms are irregular in contour, they are not amoeboid and quickly assume an oval or spherical shape.

Schizonts. The nucleus of the asexual stages divides early and the large nuclei are prominent bodies, well separated from each other $(f i g .1 \mathrm{~F}$ ). Further multiplication occurs and the nuclei tend to assume a peripheral position (fig. $1 \mathrm{G}$ ). Later the nuclei may become jammed together at one extremity and the pigment is concentrated at the narrower end, so that a fan-shaped schizont is produced (fig. $1 \mathrm{H}$ ). In early schizogony the black pigment very ofter lies on the very edge of the parasite, almost as if it were being extruded (fig. $1 \mathrm{~F}$ ). The mature schizont ( segmenter $»)$ contains 12-16 merozoites (fig. $1 I-J)$ and while still in the erythrocyte measures $4.5 \mu \mathrm{m}$. When the corpuscle disintegrates, the merozoites may spread out slightly before final separation and will then occupy a slightly larger area $(7 \mu \mathrm{m})$. The concentrated pigment may lie in the middle of the merozoites or on one side. The nucleus of the merozoite is rather irregular in shape and in staining intensity. These schizogonic stages are very common in the blood films.

Microgametocytes. The microgametocyte measures less than $7 \mu \mathrm{m}$ and is slightly larger than the uninfected corpuscle. The cytoplasm is pale and the nucleus is diffuse, spreading out over much of the parasite in a pink-staining network with one or two 
darker chromatin masses (fig. $1 \mathrm{~K}$ ). The pigment assumes different forms, but is often in 5 or 6 large, circular grains, separated from each other; sometimes it is in the form of two jagged black masses.

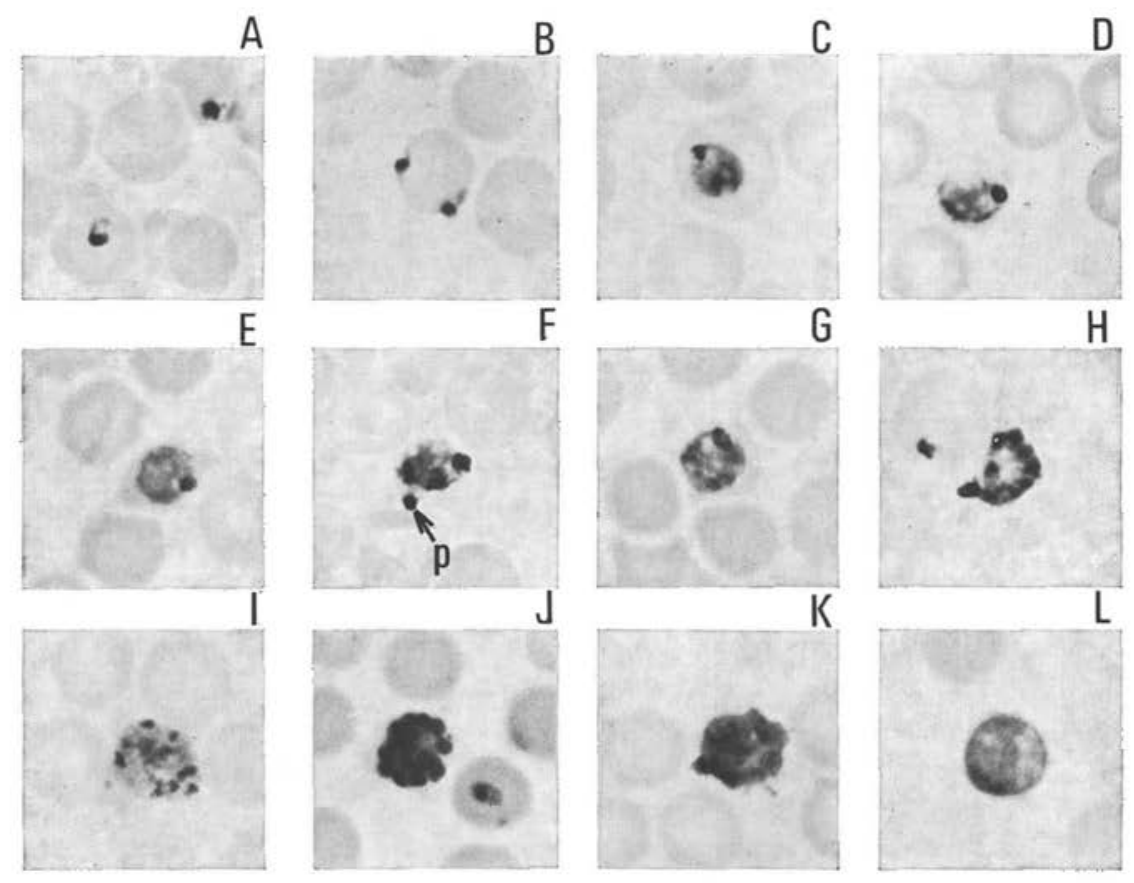

FIG. 1. - Plasmodium girardi. A : youngest forms; B : double accolé infection; C: trophozoice with pigment; D: trophozoites; E : trophozoite in « ovale » cell; F : immature schizont with 3 nuclei and pigment $(\mathrm{p}) ; \mathrm{G}$ : schizont in distorted cell; $\mathrm{H}$ : fan-shaped schizont and «ring»; I : maturing schizont; $\mathbf{J}$ : schizont with merozoites ard « ring ; $\mathrm{K}$ : microgametocyte; L : macrogametocyte. Giemsa-saline blood films, $\times 1,500$.

Macrogametocytes. The females (fig. $1 \mathrm{~L}$ ) were commoner than the males in our material, and both immature and mature forms were oval or spherical bodies, staining blue with a dense nucleus, usually centrally placed. The pigment was concentrated into a single mass.

Effect on Infected Erythrocyte. The asexual parasite produces no enlargement of the corpuscle, though the gametocytes are usually slightly larger than the uninfected corpuscle which measures 5.0-6.0 $\mathrm{m}$ in diameter. Some pallor of the erythrocyte eventually occurs, but no stippling is seen. The infected cell, however, becomes distorted in various ways : it may assume an «ovale» shape (fig. $1 \mathrm{E}$ ), or have a starfish contour (fig. $1 \mathrm{G}$ ), or it may become so damaged that only traces of the disintegrating envelope can be discerned on one side: the parasite in these conditions often becomes extracellular. 
The chief differences between our description of $P$. girardi and that of the original authors are shown in Table $I$.

TABLE 1. - P. girardi. Morphological differences in two strains.
Character
Bück et al.
Garnham and Uilenberg

Vacuole of trophozoite $\ldots \ldots$ Bien marquée $\ldots \ldots \ldots \ldots \ldots$ Usually rather inconspicuous.

Number of merozoites ...... 8-10 (exceptionnellement (12 ?) . 12-20.

Pigment in mature schizont ... Rare au centre .......... Often central.

Decolourization of erythrocyte. Absent .............. Present.

Relationship to other species of

Plasmodium in primates.

Type Quartan..$\ldots \ldots \ldots \ldots$ No evidence of quartan periodicity.

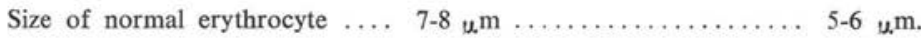

\section{Plasmodium foleyi Bück et al., 1952}

This parasite was described from the blood of a lemur ( $L$. fulvus rufus $=L$. macaco rufus) which had been splenectomised 19 days previously and had suffered a relapse of $P$. girardi 12 days after splenectomy. There was thus a parasitaemia comprising the two species after $P$. girardi had run its course alone for a week. The characters of $P$. foleyi are shown in Table $I I$; the three principal distinctive features were great enlargement of the infected corpuscle, the late appearance of fine brown pigment, and the absence of mature schizonts and gametocytes.

No original material was preserved.

\section{Plasmodium lemuris Huff and Hoogstraal, 1963}

A single blood film of Lemur collaris $(=L$. macaco collaris) was found to contain 30 malaria parasites which the authors thought represented a different species from $P$. girardi or $P$. foleyi and named it $P$. lemuris. The animal had not been splenectomised. The characters of $P$. lemuris are shown in Table II, and the principal diagnostic features are the enlargement of infected cells and the gross enlargement of gametocytes, the absence of mature schizonts, and brown pigment.

The descriptions of both $P$. foley $i$ and $P$. lemuris are unaccompanied by measurements of the parasites ; also the scale of the figures does not correspond to the descriptions ( $P$. foleyi is said to make the corpuscles «très augmentées de volume »; the $P$. lemuris plate shows uninfected corpuscles as $10-11 \mu \mathrm{m}$ in diameter according to the magnification of $1000 \times$ - actually the erythrocytes of $L$. macaco measure 5-6 $\mu \mathrm{m}$ in diameter and it is obvious that the magnification should be doubled, i.e. $\times 2000$ ). 
If the latter scale is applied it will be found that the gametocytes of P. lemuris as illustrated in the plate are about $11 \mu \mathrm{m}$ in average diameter; this is the identical figure that we give for the parasite, Plasmodium sp. Uilenberg (1970). Dr. Christine Hawkey has kindly allowed us to quote her figure of $6 \mu \mathrm{m}$ as the diameter of erythrocytes of lemurs in stained blood films.

\section{Plasmodium sp.}

Uilenberg (1970) reported the appearance of a malaria parasite, identified at that time as $P$. lemuris, in a Lemur macaco fulvus 21 days after splenectomy, and 14 days after $P$. girardi had also appeared in its blood. He stated that these two parasites would be fully redescribed later.

Fortunately the two species are readily distinguishable in the blood and we had no difficulty in following their separate courses over several weeks.

Early Forms. The very youngest parasites cause no enlargement of the erythrocyte, but immediately the parasite begins to grow, the corpuscle enlarges (fig. $2 \mathrm{~A}-\mathrm{B}-\mathrm{C}$ ) and very soon the characteristic stippling becomes visible. The central vacuole, so much a feature of «malaria rings », is practically absent, though a small vacuole may form close to the nucleus (fig. $2 \mathrm{~A}$ ). The nucleus itself is large and diffuse, often rather irregular in outline. When the parasite is about $5 \mu \mathrm{m}$, an inconspicuous dot of brown pigment may be found in the cytoplasm. Occasionally double infections of the erythrocytes occur (fig. $2 \mathrm{D}$ ). These parasites are not very amoeboid. Rarely the nucleus is divided into two or more fragments.

Immature Schizonts. As the parasite grows, the cytoplasm becomes more dense and stains a deep blue colour. The nucleus divides into two (fig. $2 \mathrm{E}$ ) and subsequent divisions are remarkable in their indication of the intense activity in the nucleus. Thus the nuclei exhibit bizarre shapes and irregular contours (fig. $2 \mathrm{H}$ ). Schizonts with three to six nuclei (fig. $2 F-G$ ) were seen in our material, but no more, and it is very probable that schizogony, as in certain other species of Plasmodium, is completed in the internal organs. It seems likely that the total number of merozoites is likely to be high, for the nuclei of the oldest stages in the peripheral blood are very large, chunky objects. Often one nucleus is larger than the others (fig. 2 E) and is undergoing division ; in other examples the nucleus is «peppered » with tiny dots of chromatin - possibly the separated mitotic elements. Another feature is the straight border on at least one side of the parasite (fig. $2 \mathrm{~J}$ ); on the other hand, completely circular parasites (fig. $2 G$ ) are quite common. Pigment is inconspicuous in the immature schizonts and rapidly fades in old blood films. Stippling of the infected corpuscle (fig. $2 L$ ) continues to be a characteristic feature and is more of the Ziemann's than the Schüffner's type. The size of the immature schizonts is from $8-11 \mu \mathrm{m}$ and the corpuscle measures about $10-13 \mu \mathrm{m}$ in diameter.

Gametocytes. It is difficult to identify the younger sexual stages, though the character of the pigment offers a clue: the grains are more abundant and do not 
clump as in the asexual forms. The nucleus of the microgametozyte occupies a large part of the body of the parasite and stains a deep red colour while the cytoplasm is pink (fig. $2 I$ ) ; a centriole is often visible just beyond the nuclear membrane. The macrogametocyte (fig. $2 J-K-L$ ) has an even more dense nucleus which may have a
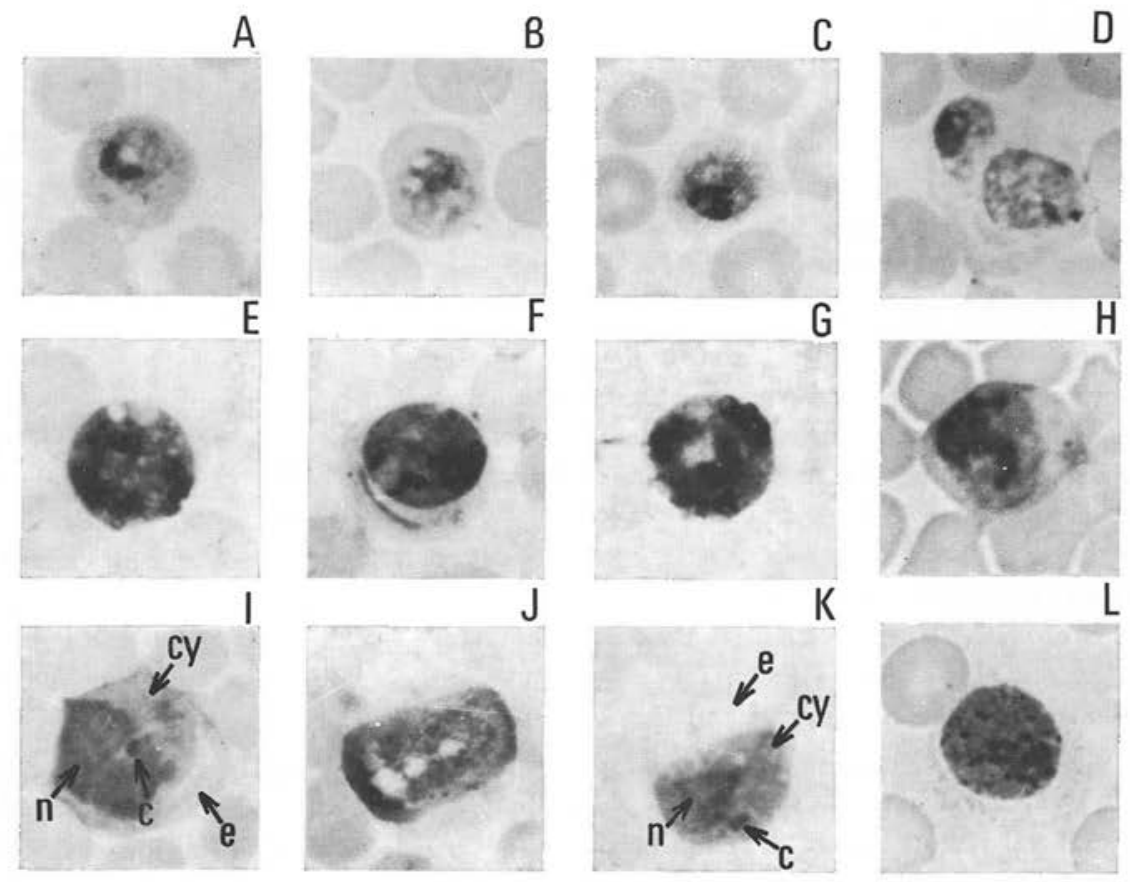

FIG. 2. - Plasmodium foleyi. A : large «ring» in Ziemann-stippled cell; B : vacuolated trophozoite; C: trophozoite with large nucleus; D: double infection with trophozoites; $\mathrm{E}$ : schizont with one nucleus and another in process of division; $\mathrm{F}$ : schizont with 3 nuclei ; G: schizont with 8 nuclei; $\mathrm{H}$ : asexual form; I: mature male showing centriole (c), nucleus (n), cytoplasm (cy) and erythrocyte (e); J : gametocyte with straight borders; K : macrogametocyte showing centriole (c), nucleus (n), cytoplasm (cy) and erythrocyte (e); L: macrogametocyte in Ziemann-stippled and enlarged cell. Giemsa-saline blood films, $\times 1,500$.

much convoluted border. In both sexes, greenish-blue staining « volutin granules may be present in the cytoplasm, usually in a small patch. The gametocytes grow to $11 \mu \mathrm{m}$ in diameter and the infected corpuscle may reach a size of $14 \mu \mathrm{m}$. The corpuscle is well stippled, and the granulations may be condensed by pressure into a deeply staining border to the corpuscle.

This malaria parasite was seen in one splenectomised specimen of L. macaco fulvus, but not in L. m. macaco. L. mongoz mongoz showed a fleeting parasitaemia only after subinoculations of infected blood. 


\section{Discussion}

Although there are minor differences between the original description of $P$. girardi and our own, there is no justification for regarding them as separate species (see p. 412).

A more difficult situation arises in regard to the other three parasites and it is necessary to realise that the circumstances were different in each. Thus, the staining quality of the material studied by Bück et al. appears to have bzen rather poor, that of Huff and Hoogstraal moderate and that of our own optimum, for we used the Giemsa saline meihod of Shute and Maryon (1966) which enhances stippling of the corpuscle; the hosts were different; the $P$. lemuris-infected lemur had an intact spleen, while the other observations were made on splenectomised animals. Nevertheless, if the descriptions are carefully analysed, it will be seen (Table II) that the most important features are common to them all. The similarities between the three parasites compared in Table $I I$ are as follows :

The parasites were all detected first as very low-grade infections in Lemur macaco (in the subspecies rufus, fulvus and collaris). Only immature schizonts of $P$. foleyi, of $P$. lemuris and Plasmodium sp. were found in the peripheral blood; these forms are badly described and illustrated by the authors of $P$. foleyi, but the structure of the nucleus in the other two parasites was found to be strikingly similar: the nucleus had a large irregular shape and was often continuous i.e. not clearly separated. The inconspicuous pigment and its light brown colour is characteristic of them all.

Bück et al. refer to the «benign tertian » type of the schizont of $P$. foleyi, presumably on account of its large size and the hypertrophy and pallor which it induces in the erythrocyte. We hardly accept the designation «benign tertian » but all three parasites are highly characterised by the gross and early enlargement of the infected corpuscle. Ziemann's dots (or Ziemann-like dots) are difficult to elicit, and we agree that the stippling obzerved by us was not of the Schüffner's pattern. With poor staining, the only feature obtainable is pallor of the corpuscle, on which Bück et al. comment. Huff and Hoogstraal made a significant observation about a pink rim of erythrocyte around the gametocyte and this is likely to be the compressed remnants of a light stippling in the corpuscle.

The apparent absence of gametocytes of $P$. foleyi was probably due to blood films not having been taken by Bück et al. sufficiently late in the infection. The spectacular appearance of these forms by Huff and Hoogstraal and ourselves is again confirmation that we were dealing with the same parasite.

Features such as amoeboidicity may vary according to the speed of drying the blood film; the presence of « rings » (or even «schizonts ») may be a question of terminology ; the colour of malaria pigment is affected by the illumination, and its persistence in old films by the quality of preservation.

Garnham (1966) placed $P$. foleyi in the group incertae sedis and suggested that it might be a species of Hepatccystis. Against this idea is the behaviour of the parasite 


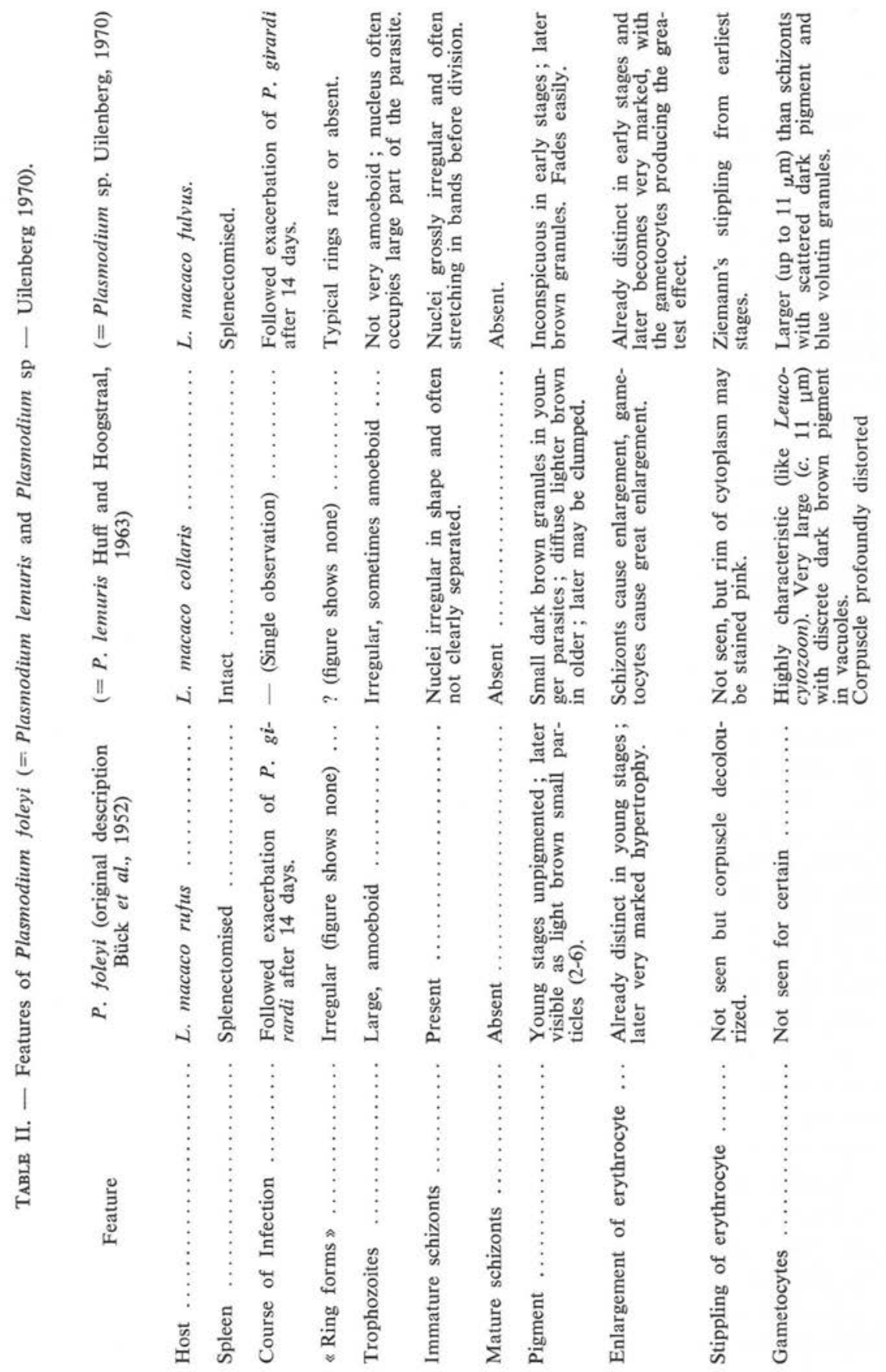


after splenectomy ; before the operation, $P$. foleyi was not seen, but it appeared in the blood 19 days later and increased in density on the following day. Hepatocystis spp. are unaffected by removal of the spleen and the relapse of the parasite on this occasion indicates that it was probably a species of Plasmodium. Huff and Hoogstraal considered that $P$. foleyi has uncertain status because no gametocytes were described (but neither were exoerythrocytic schizonts or sporogonic stages); the absence of a certain stage of the life cycle does not justify ignoring a named species, however inadequately described.

We feel therefore that the three parasites compared in Table II are one and the same. The earliest name is Plasmodium foleyi Bück et al., 1952 and Plasmodium lemuris Huff and Hoogstraal, 1963 is here placed as a synonym.

Uilenberg (1970) mentioned briefly the occurrence of Plasmodium sp. in the blood of an Avahi laniger, a lemur belonging to the family Indridae. Only autopsy material was available and no identification to species was possible, but the absence of enlargement of the infected erythrocytes excludes the diagnosis of $P$. foleyi.

\section{Revision of Plasmodium girardi and Plasmodium foleyi}

\section{Plasmodium girardi}

Bück, Coudurier and Quesnel, 1952 ; Garnham and Uilenberg, emend., 1975.

Diagnosis (blood stages). - Vacuole of young forms inconspicuous; 12-16 merozoites in schizont; heavy pigment often on edge of parasite; infected corpuscle decolourized and distorted but not enlarged or stippled; gametocytes slightly larger than uninfected erythrocyte and with coarse black pigment.

Type host ......................... Lemur macaco rufus.

Additional hosts ................... Lemur macaco macaco and L. m. fulvus.

Laboratory host ................. Lemur mongoz mongoz.

Geographical distribution ............... Madagascar.

Neo-syntypes ...................... Deposited in Wellcome Museum of Medical Science, London.

\section{Plasmodium foleyi}

Bück, Coudurier and Quesnel, 1952; Garnham and Uilenberg, emend., 1975. Syn. P. lemuris Huff and Hoogstraal, 1963.

Diagnosis (blood stages). - Typical ring forms rare or absent ; trophozoites large and sometimes amoeboid; only immature schizonts (with up to 6 massive and some- 
times linking nuclei) present in peripheral blood ; pigment absent in young forms, but later present as a few light brown granules ; infected cell is very greatly enlarged and becomes lightly stippled ( Ziemann's dots »); gametocytes may be $11 \mu \mathrm{m}$ in diameter and contain scattered pigment and «volutin» granules.

Type host ........................ Lemur macaco rufus.

Additional hosts ..................... Lemur macaco collaris and L. m. fulvus.

Geographical distribution $\ldots \ldots \ldots \ldots \ldots$ Madagascar.

Neo-syntypes ..................... Deposited in Wellcome Museum of Medical Science, London.

\section{Bibliography}

Bück (G.), Coudurier (J.) et Quesnel (J.-J.), 1952. - Sur deux nouveaux Plasmodium observés chez un lémurien de Madagascar splénectomisé. Arch. Inst. Pasteur Algér., $30,240-243$.

Garnh 4M (P. C. C.), 1966. - Malaria parasites and other Haemosporidia. Blackwell Scientific Publications, Oxford, 1114 p.

Huff (C. G.) et Hoogstraal (H.), 1963. - Plasmodium lemuris n. sp. from Lemur collaris E. Geoffroy. J. infect. Dis., 112, 233-236.

Shute (P. G.) et MARYon (M.), 1966. - Laboratory technique for the study of malaria. 2nd edition. J. and A. Churchill Ltd., Publ., London, 112 p.

Uilenberg (G.), 1970. - Quelques Protozoaires parasites de Mammifères sauvages à Madagascar. Ann. Parasit. hum. comp., 45, 183-194. 